\title{
Experience-Dependent Plasticity from Eye Opening Enables Lasting, Visual Cortex-Dependent Enhancement of Motion Vision
}

\author{
Glen T. Prusky, ${ }^{1,2}$ Byron D. Silver, ${ }^{2}$ Wayne W. Tschetter, ${ }^{1,2}$ Nazia M. Alam, ${ }^{1,2}$ and Robert M. Douglas ${ }^{3}$ \\ ${ }^{1}$ Department of Physiology and Biophysics, Weill Cornell Medical College, Burke Medical Research Institute, White Plains, New York 10605, ${ }^{2}$ Department of \\ Neuroscience, University of Lethbridge, Lethbridge, Alberta, Canada T1K 3M4, and ${ }^{3}$ Department of Ophthalmology and Visual Sciences, University of \\ British Columbia, Vancouver, British Columbia, Canada V5Z 3N9
}

\begin{abstract}
Developmentally regulated plasticity of vision has generally been associated with "sensitive" or "critical" periods in juvenile life, wherein visual deprivation leads to loss of visual function. Here we report an enabling form of visual plasticity that commences in infant rats from eye opening, in which daily threshold testing of optokinetic tracking, amid otherwise normal visual experience, stimulates enduring, visual cortex-dependent enhancement $(>60 \%)$ of the spatial frequency threshold for tracking. The perceptual ability to use spatial frequency in discriminating between moving visual stimuli is also improved by the testing experience. The capacity for inducing enhancement is transitory and effectively limited to infancy; however, enhanced responses are not consolidated and maintained unless in-kind testing experience continues uninterrupted into juvenile life. The data show that selective visual experience from infancy can alone enable visual function. They also indicate that plasticity associated with visual deprivation may not be the only cause of developmental visual dysfunction, because we found that experientially inducing enhancement in late infancy, without subsequent reinforcement of the experience in early juvenile life, can lead to enduring loss of function.
\end{abstract}

Key words: optomotor; optokinetic; visual motion; plasticity; visual cortex; critical period

\section{Introduction}

It is generally accepted that the developing mammalian visual system progresses through "sensitive" or "critical" periods early in life, in which mature vision is facilitated through adaptive feedback from the visual environment. The experimental evidence for developmental plasticity of vision comes largely from studies of ocular dominance plasticity, in which deprivation of form vision with eyelid suture during a restricted period in juvenile life (Hubel and Wiesel, 1970; Wiesel and Hubel, 1963; Giffin and Mitchell, 1978; Prusky et al., 2000b) alters the normal maturation of circuits in visual cortex [i.e., ocular dominance (Hubel and Wiesel, 1970; Wiesel and Hubel, 1963)] and produces an enduring loss of spatial vision [amblyopia (Giffin and Mitchell, 1978)] whether one or both eyes are deprived (Prusky et al., 2000b). Other studies have extended the evidence for developmental visual plasticity beyond the realm of cortical ocular dominance and spatial vision, and into infancy (Daw et al., 1978; Harwerth et al., 1990; Smith and Trachtenberg, 2007), support-

Received May 10, 2007; revised July 13, 2008; accepted Aug. 21, 2008.

This work was supported by a Natural Sciences and Engineering Research Council of Canada Discovery grant (G.T.P.). We thank Matthew Secretan, Jacqueline Quittenbaum, Valerie Lapointe, Trevor McGill, and Jennifer Lapointe for their assistance with this study.

Correspondence should be addressed to Glen T. Prusky, Burke Medical Research Institute, 785 Mamaroneck Avenue, White Plains, NY 10605. E-mail: glp2004@med.cornell.edu.

DOI:10.1523/JNEUROSCI.1940-08.2008

Copyright $\odot 2008$ Society for Neuroscience $\quad$ 0270-6474/08/289817-11\$15.00/0 ing the notion that even early visual experience plays an important role in the acquirement of normal vision.

To date, however, reports of developmental visual plasticity have been limited to loss-of-function experiments and thus may have missed important enabling properties of visual experience during development. One way to document a true enabling role for early visual experience would be to demonstrate that a visual behavior can be improved as the result of selective visual stimulation. Traditional visual stimulation/reinforcement-based behavioral testing methodologies (i.e., perceptual measures of acuity) are not suited to this purpose, because young animals apparently lack the physical and/or cognitive ability to learn and perform the tasks as infants (Prusky et al., 2000a). In addition, spatial frequency (SF) thresholds generated in reinforcementbased tasks are typically close to sampling limitations set by visual system anatomy, thereby making it difficult to detect experientially induced enhancement of function.

Previously, we developed a virtual optokinetic system (VOS) (Prusky et al., 2004) for measuring visual thresholds of optokinetic tracking (OKT), a fundamental visual behavior that facilitates the relative stabilization of retinal images. This methodology circumvents the limitations of other behavioral testing methodologies for investigating the enabling qualities of early visual experience, because thresholds can be measured through each eye separately in untrained and freely moving laboratory animals, daily from eye opening (Douglas et al., 2005). An added advantage is that OKT SF thresholds measured in experimentally 
naive animals are lower than sampling limitations of the retina, thereby making it possible to detect experience-dependent gains and losses of function.

In the present study, we used the VOS to quantify OKT in rats daily from eye opening, and found that the testing experience itself enabled enduring, visual cortex-dependent plasticity of vision, with distinct phases of induction and consolidation.

\section{Materials and Methods}

Animals. Male and female Long-Evans rats from Charles River stock were bred and reared at the University of Lethbridge and housed on a $12 \mathrm{~h}$ light/dark schedule (lights on 7:30 A.M., lights off at 7:30 P.M.). Pups stayed with the mother until their normal age of weaning at postnatal day 21 (P21) (P1 is the day after birth), at which time they were divided into same-sex cohorts of three to six animals. Experiments on infant animals were limited to those that opened their eyes on P15. Infancy is defined as the period from eye opening at P15 to weaning at P21. Juvenile life is defined as the period from weaning at P21 to sexual maturity at $\sim$ P45. All procedures were authorized by the University of Lethbridge Animal Care Committee, which implements the standards of the Canadian Council on Animal Care.

Quantification of OKT thresholds. The methodology to measure OKT thresholds has been described in detail previously (Prusky et al., 2004, 2006; Douglas et al., 2005). In brief, a vertical sine wave grating was projected as a virtual cylinder in three-dimensional coordinate space on computer monitors (mean white $=247.604 \mathrm{~cd} / \mathrm{m}^{2}$; mean black $=0.260$ $\mathrm{cd} / \mathrm{m}^{2}$ ) arranged in a quadrangle around a testing arena (Prusky et al., 2004) (OptoMotry; CerebralMechanics). Unrestrained animals were placed on an elevated platform at the epicenter of the arena. An experimenter used a video image of the arena from above to view the animal and follow the position of its head with the aid of a computer mouse and a crosshair superimposed on the frame. The $X-Y$ positional coordinates of the crosshair centered the hub of the virtual cylinder, enabling its wall to be maintained at a constant "distance" from the animal's eyes, and thereby to fix the SF of the stimulus at the animal's viewing position. When the cylinder was rotated and the animal followed with head and neck movements that tracked the rotation, it was judged that the animal's visual system could distinguish the grating.

Assessment of full-field OKT SF and contrast thresholds. Homogeneous gray was projected on the cylinder at the beginning of each testing session. When measuring SF thresholds, the experimenter waited until the animal stopped walking or grooming, at which time gray was replaced with a low SF $[0.04-0.10$ cycles per degree $(\mathrm{c} / \mathrm{deg})]$, high-contrast sine wave grating of the same mean luminance, drifting in one direction. The animal was assessed for tracking behavior for a few seconds, and then the gray stimulus was restored. This procedure was repeated until unambiguous examples of tracking were observed. The short testing epochs reduced the possibility of the rat adapting to the stimulus, it established that each animal was capable of tracking when a salient stimulus was present, and initiating the testing at a low SF allowed each animal's OKT to be typified. The SF of the grating was then increased incrementally, with multiple tests at each SF, until it was judged that the animal no longer tracked. At low SFs, the duration of a tracking event is typically $1 \mathrm{~s}$ or more, and can subtend 10 or more degrees of arc. As the SF is increased, the duration and extent of tracking is progressively reduced until the point when no tracking is discernible, which is the threshold. The process was repeated at least three times until the highest SF that elicited tracking was established. Occasional kissing noises or finger tapping on the apparatus were used to induce animals to change their position, or to stop moving or grooming, both of which facilitated more rapid testing. Thresholds through each eye were measured independently by altering the direction of cylinder rotation, because only temporal-to-nasal motion in the visual field evokes tracking (Douglas et al., 2005). All experiments were conducted using a cylinder rotation rate of $12 \%$, which is the most favorable speed to judge OKT across varying SFs. We found that the SF threshold is constant so long as the velocity remains $<20 \%$ s (Prusky and Douglas, 2008). Lower stimulus velocities produce low head velocities, which are more difficult to discern. The supplemental Movie (avail- able at www.jneurosci.org as supplemental material) provides video examples of tracking, and the supplemental Methods (available at www.jneurosci.org as supplemental material) outline details of OKT measurement variability.

Contrast thresholds were generated using essentially the same procedures, with the exception that testing began with a grating of maximum $(\sim 100 \%)$ contrast, which was then systematically reduced until the minimum contrast to generate tracking was identified. Thresholds were measured at 8-9 SFs $(0.031,0.064,0.092,0.103,0.119,0.167,0.272,0.400$, and $0.603 \mathrm{c} / \mathrm{deg}$ ), and a contrast sensitivity function was generated by calculating at each SF a Michelson contrast using the screen luminances $(\max -\min ) /(\max +\min )$ and plotting the reciprocal of the threshold.

Rats were tested during their daylight cycle, and a complete set of SF and contrast thresholds in an animal through each eye was usually generated in 10-15 min. Whenever possible, experimenters were blind to an animal's experimental condition and previously recorded thresholds, and adjunct observers were used regularly to validate threshold assessments. Unless otherwise stated, testing on a given day involved the measurement of both SF and contrast thresholds.

Assessment of partial-field thresholds. The rat retina does not have a specialized fovea; however, rats regularly "fixate" by moving their heads to place items of interest into their binocular field, suggesting that binocular and monocular functions differ. To quantify these field-specific functions as we have in mice (Prusky et al., 2006), we measured SF thresholds within and across the monocular and binocular visual fields by restricting the arc of the drifting grating in azimuth (the remainder of the cylinder was displayed with equiluminant gray). Because the tracking movements of the rat's head and neck in response to cylinder rotation are confined to a plane parallel to the horizon, we were able to measure visual field-specific responses by limiting the angle of the stimulus. To accomplish this, we monitored the animal's gaze by tracking the orientation of its head from above with the aid of a scroll wheel on the computer mouse. The vector generated by this procedure was used by the software to maintain the grating patch at a constant angle relative to the head. Because the central $30-40^{\circ}$ of the upper portion of each visual hemifield $\left(<110^{\circ}\right.$ from midline) in rats receives input from both eyes (Heffner and Heffner, 1992), we defined the binocular zone conservatively as, and limited our stimulation to, $50^{\circ}$ straddling the midline. We also measured thresholds within the monocular zone (partial, $50^{\circ}$ centered at $65^{\circ}$ from the midline; full, $75^{\circ}$ centered at $37.5^{\circ}$ from the midline) and across both fields $\left(-25^{\circ}\right.$ to $110^{\circ}$ from the midline).

Enrichment of visual experience. We used two methods to enrich rearing conditions and visual experience. The first was to raise rats from P12 in large $(1.2 \mathrm{~m}$ long $\times 1.0 \mathrm{~m}$ wide $\times 2.5 \mathrm{~m}$ high $)$ "condominiums" within which various runways, sticks, ropes, and toys were placed for the animals to interact with (Kolb and Gibb, 1991). The second method was a modification of an open field exploratory task (Lehmann et al., 2007) to enrich the visual motion experience of animals in standard housing conditions through increased exposure to visual stimuli and self-induced visual motion. The apparatus consisted of a $2.0 \mathrm{~m}$ diameter circular wooden table painted white that was elevated $0.6 \mathrm{~m}$ above the floor. The apparatus was located in a test room with a rich array of large, stable cues of various SFs and contrasts. A litter of pups was removed from their cage and placed in the center of the table for 60 min on each day from P12 to P25. During the stimulation period, animals were periodically disbursed across the table and allowed to recongregate, and experimenters regularly moved about the room.

Visual cortex ablations. Animals were anesthetized with inhaled isoflurane (induction at $2.5-4.5 \%$, maintenance at $1-2 \%$ evaporated in $1-1.5$ $\mathrm{L} / \mathrm{min}_{2}$ ) and placed in a stereotaxic frame. A topical antibacterial ophthalmic agent (BNP) was applied to the eyes, and the top of the head was shaved and then wiped with dilute Hibitane and $70 \%$ ethanol. A midline incision was made in the scalp, the skin was resected to expose the skull, and the boundaries of striate cortex were demarcated on the skull with reference to stereotaxic coordinates (Paxinos and Watson, 1998). A dental drill was used to create a trephination over visual cortex, the dura was resected, and the cortex within the opening was aspirated down to white matter. Scalp incisions were closed with staples, the animals were injected with an analgesic (buprenorphine, $5 \mathrm{mg} / \mathrm{kg}$, s.c.), recovered on a warm 
pad, and returned to their home cage once they were alert and mobile. Behavioral testing commenced the day after surgery in animals with lesions at P14, and after $2 \mathrm{~d}$ in adult animals. Once experimental procedures were completed, rats were anesthetized and perfused with cold buffered saline followed by buffered $4 \%$ paraformaldehyde. Brains were extracted, and the dorsal surface of each brain was photographed.

Visual cortex inactivation. Visual cortex was inactivated with muscimol-releasing Elvax. Sheets of Elvax were prepared using a modification of procedures described previously (Smith et al., 1995, 2004; Prusky and Ramoa, 1999). Beads of the ethylene-vinyl acetate copolymer Elvax 40-W (a gift from DuPont) were washed for $24 \mathrm{~h}$ each in five changes of $95 \%$ alcohol, followed by five changes in $100 \%$ alcohol. They were dried overnight at $40^{\circ} \mathrm{C}$ and stored thereafter at room temperature. One hundred milligrams of the dried beads were dissolved in $1 \mathrm{ml}$ of methylene chloride, and the solution was mixed on a Vortex with $20 \mu \mathrm{l}$ of either double-distilled water (for sham implants) or an aqueous solution of muscimol. Muscimol was dissolved in one equivalent of $10 \mathrm{M} \mathrm{NaO}-$ $\mathrm{H}_{(\mathrm{aq})}$, and the volume was made up to $20 \mu \mathrm{l}$ with double-distilled water. Ten microliters of $5 \%$ fast green $_{(\mathrm{aq})}$ were then added to the mixture, and the solution was mixed on a Vortex for $10 \mathrm{~min}$. The solution was frozen on dry ice and then transferred to $-80^{\circ} \mathrm{C}$ for $6 \mathrm{~d}$ followed by another $4 \mathrm{~d}$ at $-20^{\circ} \mathrm{C}$. The resulting block of repolymerized Elvax was cut into 200 $\mu \mathrm{m}$ sheets on a freezing microtome and stored on glass slides at $4^{\circ} \mathrm{C}$. Sheets of prepared Elvax were cut into $4 \mathrm{~mm}^{2}$ squares and washed in sterile PBS for $24 \mathrm{~h}$ before surgery.

Animals were prepared for surgery as above, with the exception that a $3.0 \mathrm{~mm}^{2}$ trephination was made over the center of visual cortex in each hemisphere, and sheets of Elvax were placed on the cortical surface by tucking them under the edges of the bone. After 1-3 weeks of testing, the trephination was exposed as above, and the Elvax was removed by gently sliding it out from under the edges of the bone. Incisions in the scalp were closed as above, and postsurgical care was identical. After completion of behavioral testing, brains were extracted and prepared as above, and the cortex was inspected and digitally photographed.

Quantification of visual perceptual thresholds. We used a visual discrimination task [visual water task (VWT)] to measure grating acuity and motion sensitivity thresholds. The methodology has been described in detail previously (Prusky et al., 2000a; Douglas et al., 2005). Briefly, a trapezoidal-shaped tank is made into a Y-maze with a central divider at the wide end, and computer monitors project visual stimuli through a glass wall at the end of each arm. Water is added to the tank to a depth sufficient to submerge a platform directly below the monitor displaying a positive $(+)$ stimulus. Nothing is located below the monitor displaying a negative $(-)$ stimulus. Over a series of trials wherein the left/right position of the + and - stimuli are varied pseudorandomly, animals are trained to discriminate reliably between the stimuli by swimming to the monitor displaying the + stimulus, where they can escape from the water by mounting the platform. After training, the threshold to make the discrimination is determined by systematically reducing the difference between the + and - stimuli until animals make decisions below $7 / 10$ correct. A cumulative normal curve is fit to a plot of the data, and the point on the curve that intersects with $70 \%$ correct is adopted as the threshold. Visual stimuli were generated with, and the experiments were controlled by, custom software (Vista; CerebralMechanics) running on a G3 Apple Macintosh.

Grating acuity was measured by first training animals to discriminate a stationary, vertically oriented sine wave grating $(+)$ from gray of the same mean luminance $\left(-;\right.$ mean white $=74.013 \mathrm{~cd} / \mathrm{m}^{2}$; mean black $=$ $0.0318 \mathrm{~cd} / \mathrm{m}^{2}$ ) (Prusky et al., 2000a), and then systematically reducing the SF of the grating over a series of trials until accuracy fell below $70 \%$ correct. A threshold to discriminate the SF of gratings moving in different directions was also measured. In this case, animals were first trained to discriminate a low-SF, vertically oriented sine wave grating drifting rightward at $12 \%$ s (+) from an identical grating drifting leftward $(-)$. The SF of the gratings was then increased until the threshold ( $70 \%$ correct) to make the discrimination was identified.

A dot motion coherence threshold, in which the ability to discriminate the direction of motion in fields of dots moving in opposite directions, was generated using previously described methods (Douglas et al., 2006).
The stimuli on each monitor consisted of 24 frame movies that looped continuously during a trial. Each frame had round white dots randomly positioned on a black screen, which covered $\sim 20 \%$ of each screen. The dots had a luminance of $90 \mathrm{~cd} / \mathrm{m}^{2}$, and the background was $2 \mathrm{~cd} / \mathrm{m}^{2}$. Motion coherence, the percentage of dots moving in the same direction from one frame to another, varied between 0 and maximal coherence (92\%). Within each movie, the amplitude of the displacements from frame to frame was the same for all dots. The + stimulus consisted of coherent movement to the right, and the - stimulus had identical leftward movement. For dot motion coherence measurements, animals were trained to discriminate between the screens ( dot size $=2.98^{\circ}$; density $=20 \%$; noise type $=$ nondirectional; contrast $=100 \%$; angle $=0^{\circ}$; dot lifetime $=0.423 \mathrm{~s}$; step size $=2.653^{\circ}$; step duration $=0.035 \mathrm{~s}$ ), and then the coherence of the dots was systematically reduced until a threshold to make the discrimination was identified. The ability of animals to discriminate the size of coherently moving dots was also assessed using the procedures described above, but rather than reducing the motion coherence of the dots from maximum, the size of the dots was reduced over trials until a threshold to make the discrimination was identified.

Statistics. Time series were analyzed with repeated-measures ANOVAs (using Kaleidagraph from Synergy Software) with Tukey's honestly significant difference tests being used to test differences between two days. The F-ratios were typically very high $(>100)$ and the results highly significant. OKT thresholds were usually determinable within \pm 1 cycle $(0.0028 \mathrm{c} / \mathrm{deg})$, and the SEM for groups of littermates often were of a similar magnitude. However, an analysis of all replications of the P15 to P25 enhancement effect in 37 animals across different litters, experimenters, and versions of the apparatus suggested that a better estimate of the significiant difference of the intersubject variability was higher $(0.0112 \mathrm{c} / \mathrm{deg})$, and this more conservative estimate was used in selective $t$ tests that compared differences between groups. To correct for the multiple, nonorthogonal $t$ tests, the probability criterion for significance was set at 0.01 . Note also that SE bars, using either variance estimate, are not visible on most graphs because they are much smaller than the plot symbols, which cover approximately $\pm 0.02 \mathrm{c} / \mathrm{deg}$ in most plots.

\section{Results}

\section{Visual cortex-dependent enhancement of OKT SF threshold from eye opening}

We measured OKT thresholds daily from the onset of functional vision at eye opening (P15) and found that the SF threshold increased from $0.25 \mathrm{c} / \mathrm{deg}$ on P15 to $0.54 \mathrm{c} / \mathrm{deg}$ by P20 (Fig. $1 \mathrm{~A}$, left, filled circles), near the level of experimentally naive adults (open circle) (Douglas et al., 2005) (examples of tracking behavior are presented in the supplemental Movie, available at www. jneurosci.org as supplemental material). To our surprise, the threshold did not plateau at this value, but continued to increase until P25, reaching $0.833 \pm 0.003 \mathrm{c} / \mathrm{deg}, 55 \%$ above the baseline of naive adults $(t=54.9 ; p<0.0001 ; \mathrm{df}=21)$, and with continued testing through P30, remained there into adulthood. To determine whether it was the experience of measuring OKT itself that caused the unexpected enhancement, we divided a litter of 12 animals into two groups of six, and then measured the sensitivity of OKT daily from P15 in one group, and from P25 (the age at which developmental changes stabilized) in the other. Figure $1 \mathrm{~A}$ (right) shows that the group tested from P15 (filled circles) exhibited an enhancement profile similar to that of animals in Figure $1 A$ (left) with testing from P15, whereas animals tested from P25 (open circles) did not progress above naive adult baseline values $(t=1.7 ; p=0.11$; df $=21)$, with only a small but significant $1.4 \pm 0.4 \%$ increase afterward $\left[F_{(9,45)}=17.1 ; p<0.0001\right.$; extended daily testing after P25 did not augment this effect (supplemental Fig. 1, available at www.jneurosci.org as supplemental material)]. Thus, this second series effectively replicated the first, with daily OKT testing from P15 to P25 producing an enhancement of visual function. Contrast sensitivity also increased over 
the 2 weeks after eye opening, but the most conspicuous difference between groups tested from P15 and P25 was increased sensitivity at the highest SFs (data not shown). As a consequence, we focused our efforts on characterizing plasticity of the SF threshold and present those data here.

Previously, we reported that visual cortex (V1) lesions in experimentally naive adult rats do not reduce OKT SF thresholds (Douglas et al., 2005), supporting the generally accepted view that stabilizing retinal reflexes are normally mediated through subcortical circuits. More recently, however, we reported that the visual cortex becomes involved in an experience-dependent enhancement of the SF threshold for OKT in the adult mouse (Prusky et al., 2006), and we hypothesized that visual cortex may also be required for the developmental enhancement of rat OKT. We directly tested this by removing visual cortex bilaterally on P14, before commencing with testing OKT at $\mathrm{P} 15$. Figure $1 B$ (left) shows that the OKT SF threshold in six lesioned animals (open circles) on P15 was comparable to that of intact animals (filled circles), but instead of progressing to enhanced values with daily testing, thresholds plateaued near that of animals without P15-P25 testing experience (Fig. 1A) $(t=0.35 ; p=0.73$; $\mathrm{df}=10)$. The SF threshold of control animals lesioned at P14, but not tested until P25, also did not differ from baseline $(t=0.66 ; p=0.53$; df $=$ 8 ; data not shown). Reconstructions of the lesions revealed that aspirations effectively targeted V1 (Fig. $1 B$, right).

We further investigated the role of $\mathrm{V} 1$ in the enhancement of the OKT SF threshold by combining daily OKT testing with inactivation of visual cortex with muscimol-Elvax from P14 $(n=4)$ (Fig. $1 C)$. Figure $1 C$ (left) shows that the treatment blocked the characteristic enhancement of OKT SF sensitivity and resulted in a developmental profile similar to that in P14lesioned animals (Fig. 1B). Mechanical disruption of the cortex did not account for the blockade, because animals with sham surgery [implantation of Elvax without muscimol (Sham); $n=2$ ] followed a characteristic profile of enhancement, and the cortex showed no evidence of damage (Fig. 1C, right; supplemental Fig. 2, available at www.jneurosci. org as supplemental material). Collectively, the results of the ablation and inactivation experiments showed that the experiencedependent enhancement of rat OKT from eye opening depends on the presence of, and activity in, visual cortex.

\section{Experience-dependent enhancement}

The data in Figure 1 revealed that a period of plasticity was present in rats from P15 to P25, during which the experience of
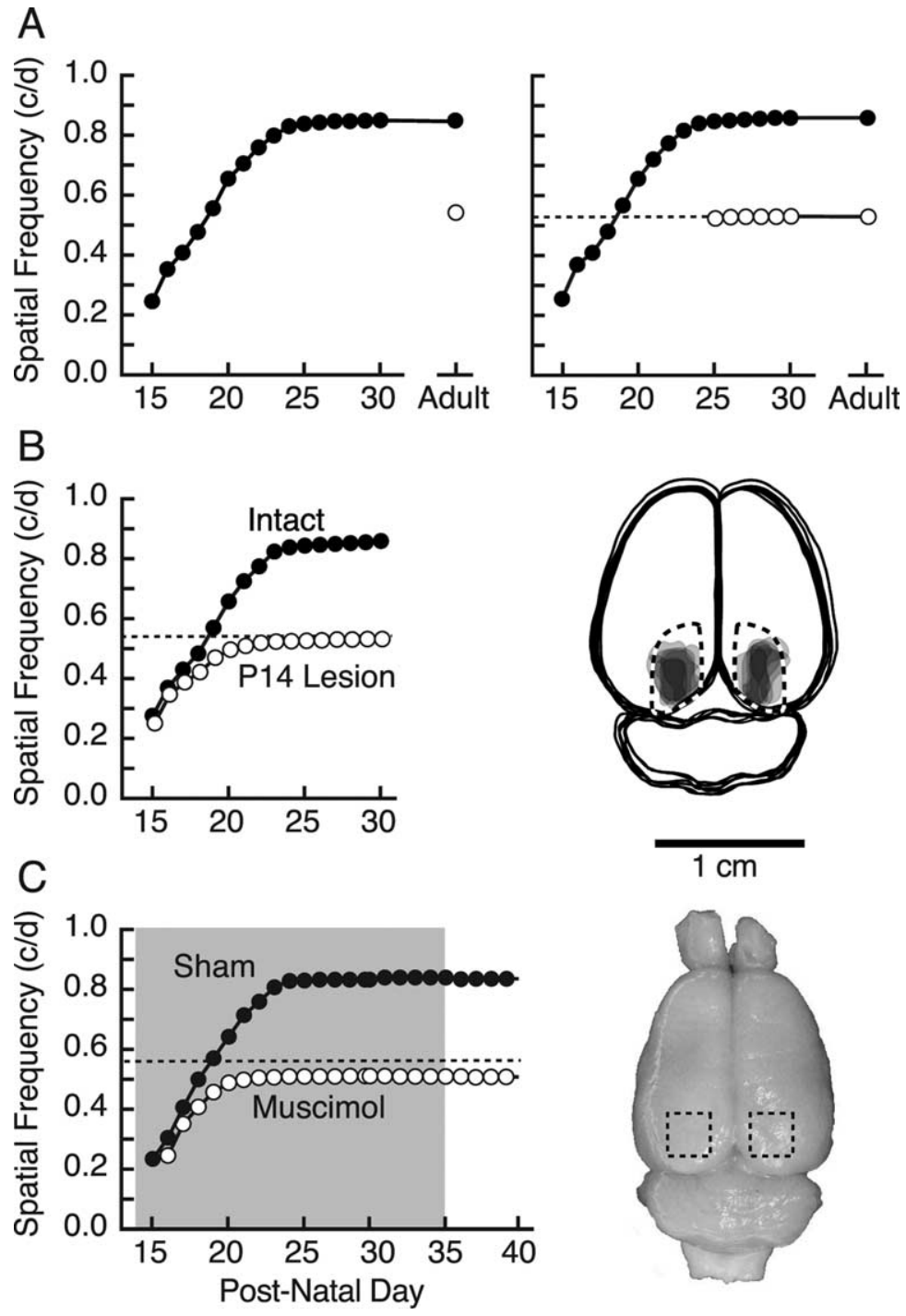

Figure 1. Visual cortex-dependent enhancement of rat OKT from eye opening. $\boldsymbol{A}$, Left, OKT SF threshold was low but measurable on P15 (filled circles) and by P20 increased to near the level of experimentally naive animals (open circle) (Douglas et al., 2005). Thresholds continued to increase until plateauing near P24-P25 and remained stable with repeated daily testing up to P30 and intermittent testing into adulthood. Right, Results of testing from P15 (filled circles) effectively replicated those in the left panel, whereas the thresholds of littermates with testing from P25 (open circles) did not differ from naive adults. The dotted line in this and other panels demarcates the OKT SF threshold of experimentally naive adult animals (open circle) in the left panel. $\boldsymbol{B}$, Left, Bilateral aspiration of visual cortex on P14 (open circles; P14 Lesion) blocked the characteristic progression of OKT enhancement present in intact animals tested from P15 (filled circles; Intact). Right, Reconstruction of lesions in the P14 Lesion group from the left panel. Surface features of the brains and boundaries of lesions for each animal were traced in Adobe Illustrator, the area of individual lesions was filled with light gray, and the images were superimposed. Fiducial landmarks were used to estimate the borders of striate cortex (dashed lines) with reference to stereotaxic coordinates. C, Muscimol-Elvax treatment of visual cortex (period of implantation indicated with shading) blocked enhancement (open circles; Muscimol), resulting in a profile similar to that in animals with P14 lesions ( $\boldsymbol{B}$, left). Sham Elvax surgery did not affect enhancement (filled circles; Sham). Removal of Elvax had no effect on either group. Right, Surface view of a brain from a representative muscimol-treated animal in the left panel. The Elvax (approximate position demarcated with dashed box) caused no apparent tissue trauma (for additional examples, see supplemental Fig. 2, available at www.jneurosci.org as supplemental material).

measuring OKT thresholds stimulated a visual cortex-dependent enhancement of OKT. To differentiate testing-induced enhancement of OKT from the "normal" development of OKT, we "probe" tested animals on single days from P15 to P25 (i.e., tested OKT only on a given day without further testing until P25, when consecutive daily testing commenced until P30) (Fig. 2A). We found that the maturation profile of the OKT SF threshold generated in this manner did not differ from that in animals tested from P15 without a visual cortex $(t=1.9 ; p=0.10 ; \mathrm{df}=7)$, but 
A
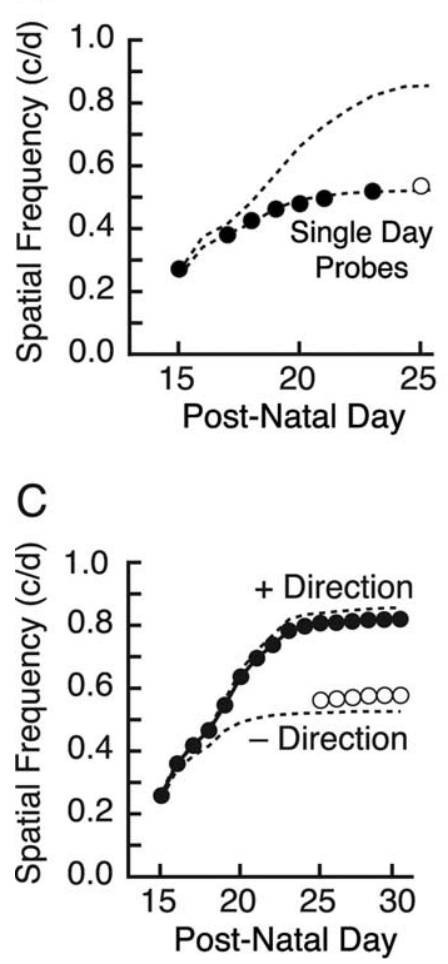

B

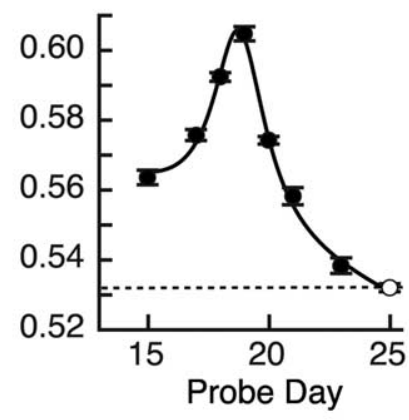

D

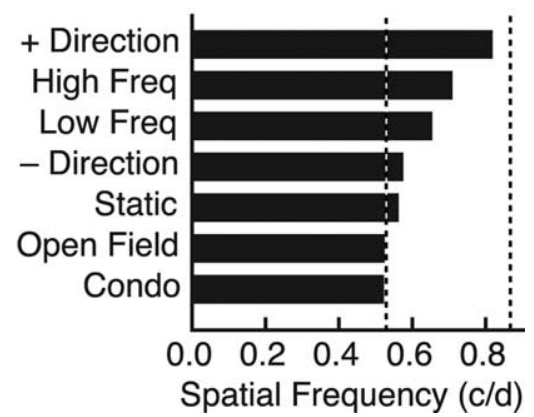

Figure 2. Experiential control of enhancement. $\boldsymbol{A}$, "Probe" measurement of OKT maturation with testing on individual days from P15 to P23 (filled circles). The open circle is the OKT SF threshold for the animals in Figure $1 A$ first tested on P25. The top dotted line is the average of the enhanced profiles in Figure 1; the bottom dotted line is the trace of P14 lesioned animals from Figure $1 C$, which is replotted here and on other figure panels as a convenient reference. $\boldsymbol{B}$, Effect on OKT SF thresholds at P25 as the result of single-day probe testing in $\boldsymbol{A}$. Fitting the data with a Lorenz peak on a linear background resolved a vertex at $18.8 \mathrm{~d}$ (black line). C, Effect on OKT SF threshold of measuring OKT from P15 to P25 in one direction (filled circles). Testing in the other direction started at P25 (open circles). D, Histogram of OKT SF threshold on P25 of P15-P24 experiential control experiments. Only experience in which animals were presented repeatedly with moving gratings that elicited tracking generated maximal enhancement. The + and - directions are replotted from $\boldsymbol{C}$. See Results for description of High Freq, Low Freq, Static, Open Field, and Condo. Dashed lines are the average adult SF thresholds for the P15 $\rightarrow$ (right) and P25 $\rightarrow$ (left) groups, which are replotted on other figure panels as a convenient reference.

it was distinctly different $(t=4.0 ; p=0.005 ; \mathrm{df}=7)$ from that produced with cumulative testing from P15 to P25 with the cortex intact; SF values progressed in both groups from P15, but the probe-tested animals did not increase as rapidly and plateaued near the same threshold $(\sim 0.53 \mathrm{c} / \mathrm{deg})$ as animals that experienced testing only from P25. These data show that the SF threshold of OKT developed gradually from P15 to P25 in the absence of OKT testing experience, and that OKT testing augmented this process; both happen together over the same period, but each has a unique temporal profile (supplemental Fig. 3, available at www.jneurosci.org as supplemental material). We also assessed the effect of the single-day testing presented in Figure $2 \mathrm{~A}$ on thresholds at P25 (Fig. 2B) and found that testing OKT on days up to P19 generated progressively greater enhancement, and regressively less enhancement thereafter, indicating that the efficacy of the plasticity is skewed toward younger ages [a graphical summary of OKT development in "enhanced" and "normal" animals is presented in supplemental Fig. 3 (available at www. jneurosci.org as supplemental material)].

We investigated the experiential specificity of OKT enhancement from eye opening by varying elements of the OKT testing experience from P15 to P25 and measuring OKT thresholds thereafter. As reported previously (Douglas et al., 2005), clockwise rotation of the grating elicits OKT only through the left eye, and counterclockwise rotation elicits OKT only through the right eye, showing that the circuitry underlying OKT is directionally biased. This bias provided us with the opportunity to carry out "within-animal" controls to investigate experiential factors of the enhancement. To do this, we measured OKT thresholds in four animals using only one direction of stimulus rotation (animals were assigned at random to leftward or rightward rotation groups) until P25, and then measured OKT thresholds in both directions thereafter. Figure $2 C$ shows that experience in the tested direction (filled circles) enabled a characteristic enhancement of the SF threshold (Fig. $1 A)$, although the plateau was at a slightly lower level $(0.819 \pm 0.0044 \mathrm{c} / \mathrm{deg})$ than in animals with equivalent experience in both directions $(0.841 \pm 0.0025 \mathrm{c} / \mathrm{deg})$ $(t=3.5 ; p=0.002 ; \mathrm{df}=19)$. When the direction of rotation opposite to the experienced direction was tested at P25 (open circles), values were only slightly higher ( 0.57 vs $0.54 \mathrm{c} / \mathrm{deg})$ than those in animals without P15-P25 testing experience $(t=$ $4.1 ; p<0.01 ; \mathrm{df}=8)$. Thus, the enhancement of OKT was effectively limited to the direction of stimulus rotation that generated tracking, even though both eyes were exposed to stimulation in both directions.

In another set of experiments, we tested whether movement of the stimulus, independent of other parameters, was necessary to enable characteristic enhancement. OKT was measured in a group of animals from P15 as in Figure 1, but the software was programmed to store the stimulus conditions for each of four animals during the testing (i.e., spatial frequency, contrast, direction of cylinder rotation, duration of stimulation, etc.). Four littermates were individually yoked to animals in the "standard" testing group, receiving the same daily experience as their yoke, with the exception that the cylinder was not rotated between P15 and P25, after which OKT thresholds were measured through both eyes. Animals in the standard testing group developed characteristic enhancement of OKT (results not shown); however, animals in the static control group were only slightly enhanced $(0.563 \pm 0.0024$ $\mathrm{c} / \mathrm{deg})$ to the same level as the opposite-direction animals $(t=$ $1.6 ; p=0.16 ; \mathrm{df}=6)$, indicating that stimulus rotation from $\mathrm{P} 15$ to P25 was necessary to induce robust enhancement (Fig. 2D, Static).

The typical testing regimen started at low frequencies, which produced strong tracking, and ended with exposure to frequencies above the threshold, which evoked no tracking. To further investigate the role of motor output (head and neck movements) in the enhancement of OKT, we stimulated a group of four animals with a grating maintained at $0.03 \mathrm{c} / \mathrm{deg}$ (slightly below the average SF threshold of OKT on P15) for $10 \mathrm{~min}$ in each direction (the approximate length of time required to measure a complete set of OKT thresholds), each day from P15 to P25, and followed with our standard testing of OKT. These animals generated a vigorous $\mathrm{OKT}$ at all ages and developed a moderate ( $20 \%)$ 
enhancement to $0.654 \pm 0.0019 \mathrm{c} / \mathrm{deg}$ (Fig. 2D, Low Freq). To test the role of visual input on its own to generate enhancement, we selected an SF $(0.83 \mathrm{c} / \mathrm{deg})$ slightly below maximal enhancement (Fig. $1 \mathrm{~A}$ ) but above the naive level, and used this to stimulate a group of four animals for $10 \mathrm{~min}$ in each direction, each day from P15 to P25. In none of the animals was a tracking response observed, yet the experience generated enhanced OKT $(0.710 \pm$ $0.0018 \mathrm{c} / \mathrm{deg}$ ) (Fig. 2D, High Freq) slightly greater $(\sim 26 \%)$ than that in the $0.03 \mathrm{c} / \mathrm{deg}$ (Low Freq)-stimulated group. Collectively, this set of experiments indicates that both sensory and motor experiences contribute to the enhancement, but neither alone is sufficient to generate maximal enhancement.

Previous developmental visual plasticity experiments have reported deprivationinduced loss of function, rather than experience-induced gains as we report here. Consequently, we were concerned that our standard housing conditions were depriving rats of "naturalistic" visual motion experience, and that OKT testing experience was replacing this deficit. We addressed this by raising nine rats in "condominium"-style housing, before commencing the measurement of OKT at P25, looking for whether these animals would be enhanced over those raised in standard housing conditions. No enhancement was detected: OKT SF thresholds were $0.522 \pm 0.0012 \mathrm{c} / \mathrm{deg}$ for this group (Fig. $2 D$, Condo). Because passively raising nocturnal rodents in enriched conditions does not guarantee that they will actually experience more visual motion, particularly during the daylight hours when we measured OKT thresholds, we provided another group of animals with augmented daytime visual motion experience from P12 to P25 in an open field adorned with salient visual cues. We then measured the effect of this experience on OKT at P25 and after. As in the condominium-raised group, open field experience did not increase OKT SF thresholds $(0.525 \pm 0.0012 \mathrm{c} / \mathrm{deg})$ (Fig. $2 D$, Open Field). Collectively, these data showed that the measurement of OKT from P15 to P25 was not merely replacing housing-induced deficiencies in visual motion experience, but that elements of the OKT testing experience itself were responsible for the enhancement.

\section{Experience- and visual cortex-dependent stabilization} of enhancement

In experiments preliminary to those in this study, we noted that enhanced responses were not always maintained, particularly in
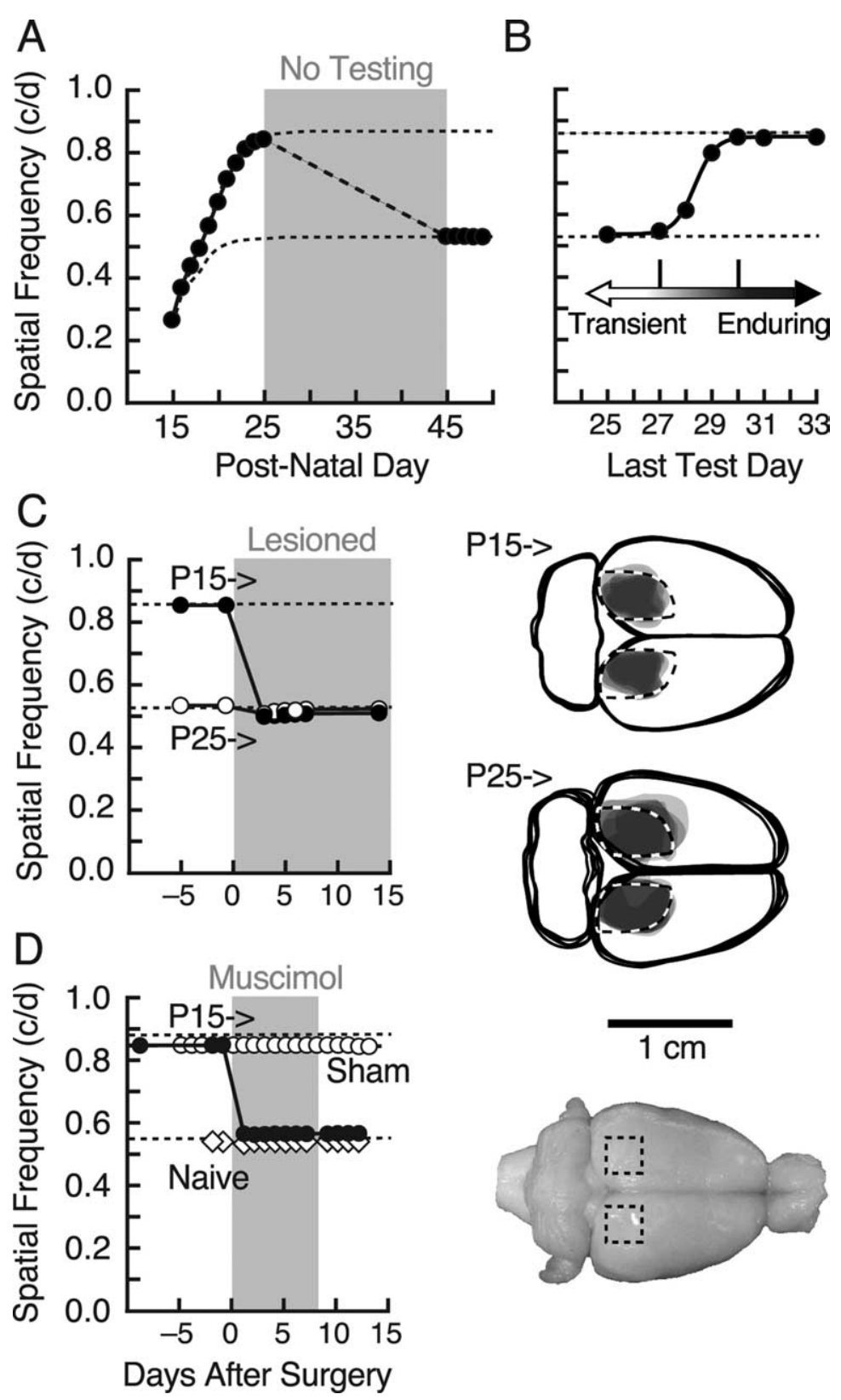

Figure 3. Visual cortex-dependent consolidation of enhancement. $\boldsymbol{A}$, Necessity of testing OKT to consolidate P15-P25 experience-dependent enhancement. Suspending testing from P25 to P45 (shading) resulted in a drop from maximal to baseline values. $\boldsymbol{B}$, Sufficiency of testing OKT after P25 to consolidate enhancement. The graph shows the effect of uninterrupted testing from P15 to P25, P27, P28, P29, P30, P31, and P33, followed by a period without testing until P45. Testing up to P27 was not able to consolidate and maintain enhanced thresholds, whereas testing thereafter was. Testing up to P28/P29 was able to rescue intermediately enhanced function. Data are fit (black line) with a sigmoidal logistic curve centered at $28.4 \mathrm{~d}$. C, Bilateral aspiration of visual cortex in animals with daily testing from P15 to P30 (P15 $\rightarrow$; filled circles) and from P25 (P25 $\rightarrow$; open circles). Thresholds in P15 $\rightarrow$ animals dropped to baseline values after the lesions, whereas thresholds in P25 $\rightarrow$ were unaffected. Right, Superimposed traces of the lesions from animals in the left panel show that lesions in both groups were centered in V1. D, Treatment with muscimol-releasing Elvax reduced thresholds of enhanced animals to near baseline (P15 $\rightarrow$; filled circles) but not in naive, unenhanced animals (Naive; open diamonds). Sham surgery in previously enhanced animals did not affect thresholds (Sham; open diamonds; $n=2$ ), nor did removal of Elvax. Right, Representative brain of a muscimol-Elvax-treated animal plotted in the left panel (see supplemental Fig. 2, available at www.jneurosci.org as supplemental material).

animals with variable daily testing after P25. We investigated this systematically using different testing regimes on animals that had developed maximal enhancement as the result of daily testing from P15 to P25. Figure $3 A$ shows that an interruption of testing for $20 \mathrm{~d}$ after P25 led to the complete loss of enhancement, decaying to naive levels $(t=0.04 ; p=0.97 ; \mathrm{df}=18)$. To characterize 

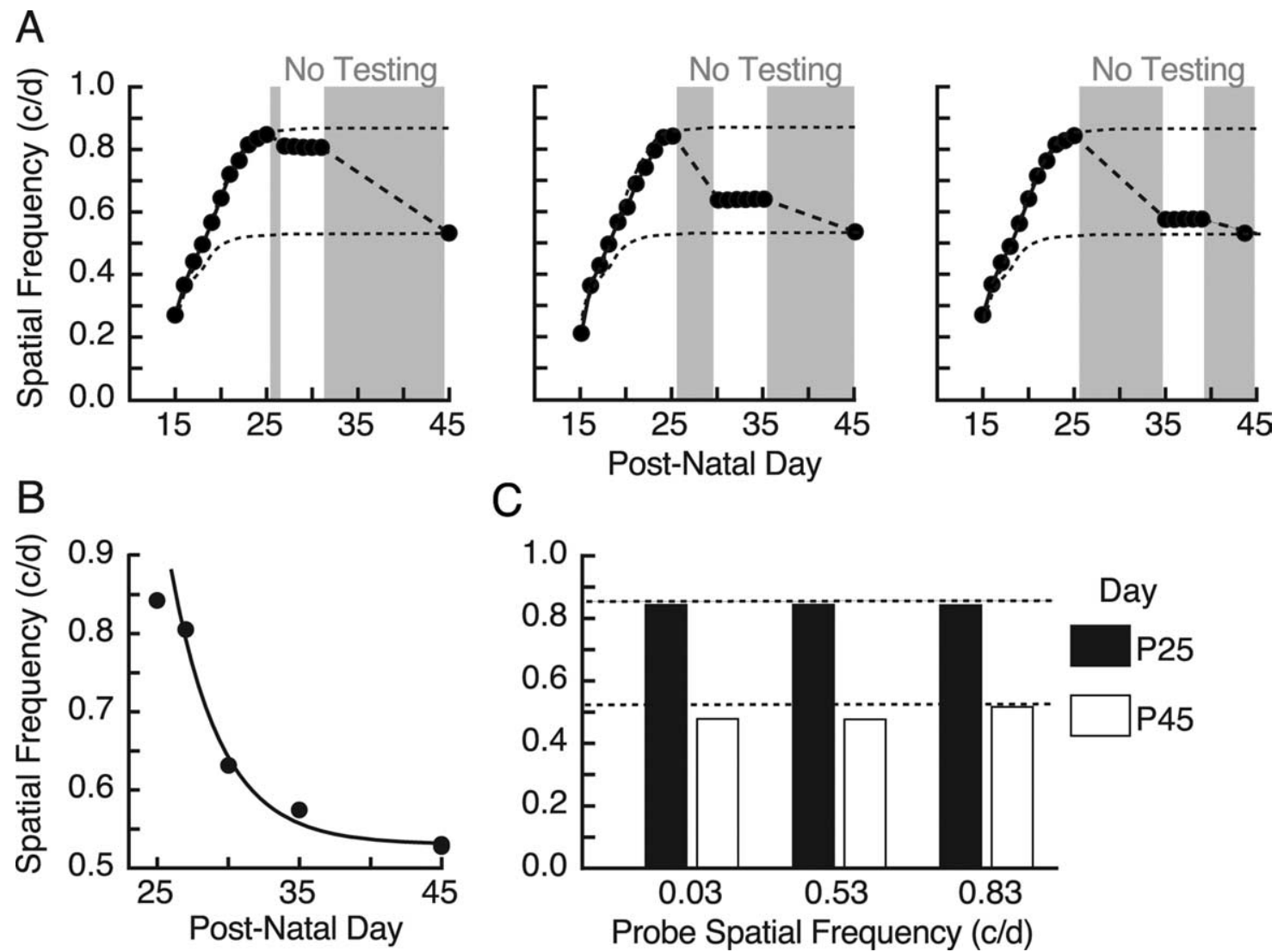

C

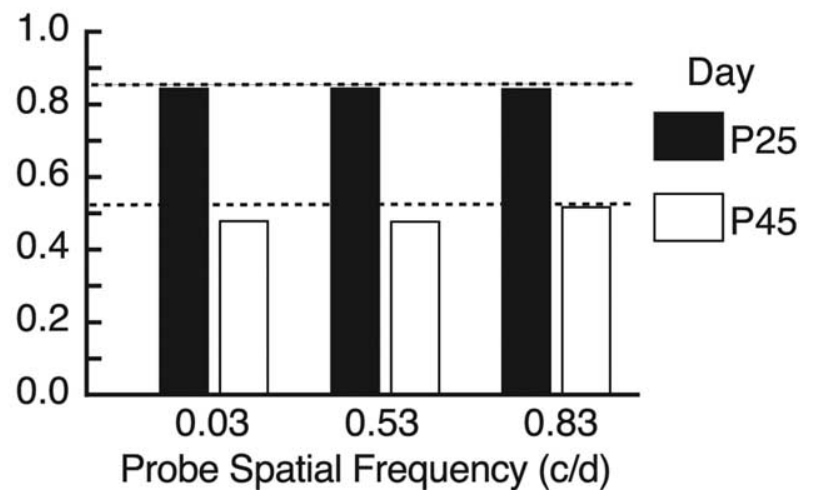

Figure 4. Experiential elements of consolidation and maintenance. $A$, Effect of a delay in testing after P25 [gray background indicates period(s) without testing] on maintenance of enhancement probed at P27 (left), P30 (middle), and P35 (right), and then measured again on P45. B, Summary of thresholds from $\boldsymbol{A}$ measured on P25, P27, P30, P33, and P45. The black line is an exponential with a time constant of $3.5 \mathrm{~d}$. The data in $\boldsymbol{A}$ and $\boldsymbol{B}$ show that any delay in testing after P25 resulted in the decay of enhancement to baseline by $\sim P 40$, which could not be rescued with repeated daily testing. C, Effect of stimulating animals with gratings of fixed SFs from P25 to P45 on enhancement generated with testing SF; contrast thresholds from P15-P25 gratings failed to maintain enhancement, with thresholds in each group falling from maximally enhanced levels at P25 (black bars) to near baseline by P45 (white bars).

the duration and experiential sufficiency of OKT testing to maintain enhanced responses, we varied the duration of testing after $\mathrm{P} 25$, and then measured responses again at P45 (young adulthood) and after. Figure $3 B$ shows that testing from P15 to P30 was sufficient to maintain enhancement indefinitely [ $>14$ months (data not shown); thresholds at $\mathrm{P} 45$ are plotted]. There was, however, a narrow window between P27 and P30 in which accumulated testing shifted the results from no enhancement, to maximal enhancement, with testing up to P28-P29 resulting in intermediate enhancement (i.e., thresholds were reduced, but they endured.). Collectively, these data established the necessity and sufficiency of testing after P25 to maintain previously enhanced responses.

To determine whether the visual cortex and its activity were necessary for maintenance of consolidated enhancement, as they were for induction (Fig. 1C,D), we aspirated V1 bilaterally in four adult animals after testing from P15 to P30 (experience sufficient to maintain maximal enhancement indefinitely) (Fig. $3 B$ ), and in six animals with testing only from P25 that were not enhanced (Fig. 1A). Figure 3C (left) shows that the lesions reduced OKT SF thresholds in enhanced animals to baseline $(t=21.7 ; p<0.0001$; $\mathrm{df}=6$; filled circles), but they had little or no effect on the threshold of animals without enhancement $(t=0.70 ; p=0.50 ; \mathrm{df}=10$; open circles). Figure $3 C$ (right) shows in overlapping reconstructions of the lesions in the P15 $\rightarrow$ group in Figure $3 C$ (left) that lesions were centered in $\mathrm{V} 1$.

We further investigated the role of visual cortex in the main- tenance of enhanced responses with muscimol inactivation (Fig. $3 D$ ). Figure $3 D$ (left) shows that inactivating V1 reduced thresholds of enhanced animals to near baseline values (filled circles; $n=3$ ), but did not reduce thresholds in naive adults [open diamonds (Naive); $n=3$ ]. Elvax implants without muscimol (Sham; $n=2$ ) did not reduce thresholds in already enhanced animals (open circles), showing that the Elvax surgery itself was not a factor in the muscimol-induced loss of enhancement. A photograph of the cortical surface of a representative muscimolElvax-treated animal in Figure $3 D$ (right) reveals no evidence of mechanical damage to the cortical surface (supplemental Fig. 2, available at www.jneurosci.org as supplemental material). Collectively, these data show that the maintenance of enhanced OKT SF thresholds, like the process of inducing enhancement, depends on the integrity of visual cortex and its activity.

\section{Iteration of infant experience in juvenile life consolidates enhancement}

Experiments in Figure 3 showed that enhancement can decay in juvenile life. To characterize this process further, we first "probed" the rate of decay by introducing variable duration interruptions in daily testing, with the first break starting on P26. Figure $4 A$ shows that the enhanced responses declined as a function (approximately exponential) (Fig. $4 B$ ) of the duration without testing. We repeated the probe test for $5 \mathrm{~d}$, the minimum duration of daily testing after P25 required to produce enduring enhancement (Fig. 3B), and found that although thresholds were 
stable during the testing, they subsequently fell to baseline, as evinced by testing at P45. These data showed that any interruption in daily testing before P30 interfered with the consolidation of enhancement and initiated a decline that could not be rescued with repeated testing.

We also evaluated characteristics of the testing experience from P25 to P30 that are necessary to consolidate and maintain enhanced function (Fig. 4C). To do this, we stimulated an enhancement of OKT with testing from P15 to P25 using our established testing procedures. We then, from P25 to P45, exposed animals for a minimum of $10 \mathrm{~min} / \mathrm{d}$ to one of three gratings of fixed SF: $0.83 \mathrm{c} / \mathrm{deg}$, an SF near maximal enhancement; $0.53 \mathrm{c} / \mathrm{deg}$, an SF near the threshold of experimentally naive adults; or $0.03 \mathrm{c} / \mathrm{deg}$, an SF all animals responded to on the day of eye opening. We followed this with regular testing at P45. Robust tracking was present in the $0.03 \mathrm{c} / \mathrm{deg}$ group throughout the stimulation period, whereas tracking was lost in the $0.53 \mathrm{c} / \mathrm{deg}$ group by $\mathrm{P} 40$, and by $\mathrm{P} 30$ in the 0.83 c/deg group. Thresholds fell from $\sim 0.84$ c/deg (black bars) to baseline or slightly below $(0.483 \pm 0.001,0.482 \pm 0.001$, and $0.518 \pm 0.001 \mathrm{c} / \mathrm{deg}$; white bars) in each of the groups. These data indicated that the testing experience used to induce enhancement in infancy (i.e., repeated measurement of OKT SF and contrast thresholds) was required in juvenile life to maintain enhancement into adulthood.

\section{Enhanced binocular field and visual motion perceptual thresholds}

Previously, we reported that OKT thresholds vary across visual fields in normal mice and in mice with enhanced OKT thresholds as the result of adult monocular deprivation (Prusky et al., 2006). To determine whether a similar trend was present in rats, we modified the OKT testing system to measure SF thresholds within restricted portions of the visual field in animals with OKT testing from P15 (enhanced, "P15 $\rightarrow$ "), and P25 (baseline, "P25 $\rightarrow$ "). As shown in Figure $5 A$ (right), in $\mathrm{P} 25 \rightarrow$ animals, the binocular-field threshold corresponded to the combined binocular/monocular threshold $(0.84 \pm 0.002$ $\mathrm{c} / \mathrm{deg})$, and the monocular-field threshold was slightly lower $\left(50^{\circ}\right.$ $\left.=0.46 \pm 0.008 \mathrm{c} / \mathrm{deg} ; 75^{\circ}=0.48 \pm 0.001 \mathrm{c} / \mathrm{deg}\right)$. The same measurements in $\mathrm{P} 15 \rightarrow$ animals (Fig. $5 A$, left) revealed that OKT SF thresholds within the monocular field were only slightly elevated $(0.54 \pm 0.005 \mathrm{c} / \mathrm{deg})$, but that sensitivity within the binocular field $(0.84 \pm 0.001 \mathrm{c} / \mathrm{deg})$, or when monocular- and binocular-field stimulation was combined $(0.84 \pm 0.001 \mathrm{c} / \mathrm{deg})$, corresponded to the enhanced responses recorded with full-field testing. These data showed that the OKT SF threshold is normally higher in the binocular field, and that testing full-field OKT from P15 exaggerated this bias.

We evaluated whether enhancement was present in visual functions other than OKT by measuring in the VWT four visual

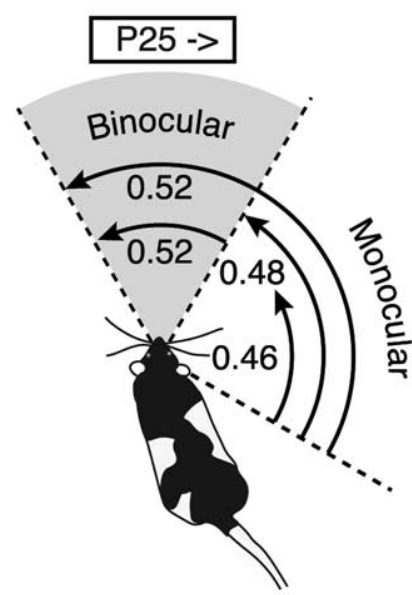

$\mathrm{P} 15 \rightarrow \mathrm{P} 25->$

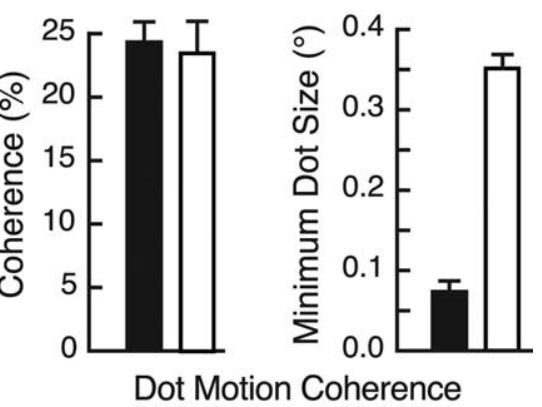

Dot Motion Coherence

Grating Acuity

Figure 5. Visual field and visual perceptual thresholds in animals with enduring enhancement. $A$, Visual field-selective OKT SF thresholds in schematic illustrations. The length of arching arrows represents the visual field segment in which the grating was present during the measurement of OKT (binocular and monocular fields delimited by dotted lines radiating from the head of the . Combined monocular-and binocular-field stimulation in animals tested from P25 (right) resulted in a threshold of $0.52 \mathrm{c} / \mathrm{deg}$. $0.48 \mathrm{c} / \mathrm{deg}$ ) regardless of the width of the stimulus within the field. Combined monocular- and binocular-field stimulation in rats (black bar) and P25 (white bar). Animals with 0KT testing experience from P15 were superior at discriminating the SF of gratings drifting at $12^{\circ} / \mathrm{s}$. Right, The dot motion coherence threshold to discriminate between kinematograms consisting of large $\left(2.98^{\circ}\right)$ dots did not differ between the groups, but animals with OKT testing experience from P15 were able to discriminate between fields of smaller dots moving at maximum coherence.

discrimination (perceptual) thresholds in the groups illustrated in Figure $1 A$ (right). Figure $5 B$ (left) shows that grating acuity (measured with stationary stimuli) of the two groups did not differ $(t=0.17 ; p=0.88$; $\mathrm{df}=5)$ and corresponded with previously published values $(\sim 1.0 \mathrm{c} / \mathrm{deg})$ (Prusky et al., 2000a; McGill et al., 2004). However, when gratings were moved at $12 \%$, the enhanced $\mathrm{P} 15 \rightarrow$ animals were able to discriminate rightward from leftward directions at a significantly higher SF $(t=5.6 ; p=$ 0.002 ; $\mathrm{df}=5$ ) than unenhanced, P25 $\rightarrow$ animals. This indicated that although the VWT acuity of P15 $\rightarrow$ animals was not enhanced, they were specifically augmented in the SF thresholds to discriminate moving stimuli. We explored this possibility by measuring the ability of the animals to discriminate the direction of movement of partially coherent random-dot patterns, because these stimuli enable the assessment of visual motion perception in the absence of positional or form cues. We found that the groups did not differ $(\sim 23 \%)$ (Fig. $5 B$, right) in the minimum dot coherence when the dots were large $\left(2.98^{\circ}\right)(t=0.60 ; p=$ 0.57 ; $\mathrm{df}=5)$. However, when we systematically reduced the size (SF) of the dots moving at maximum coherence, we found that 

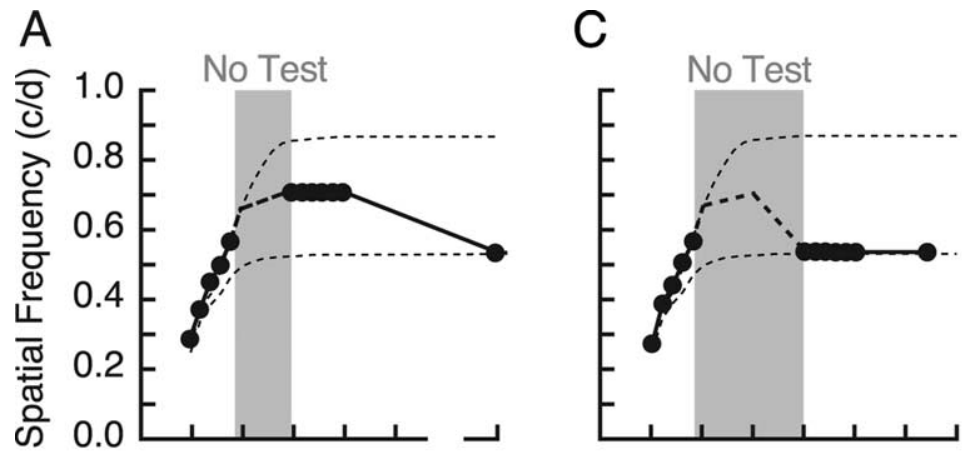

$B$

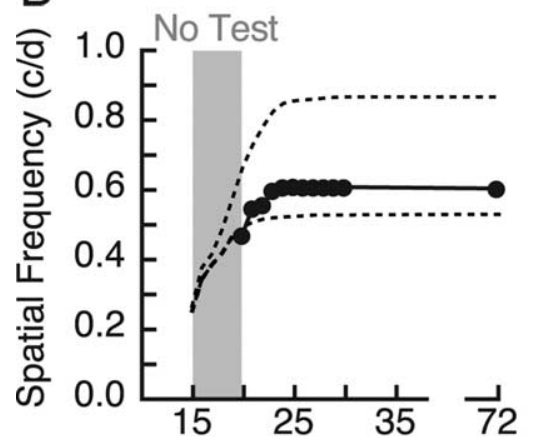

D

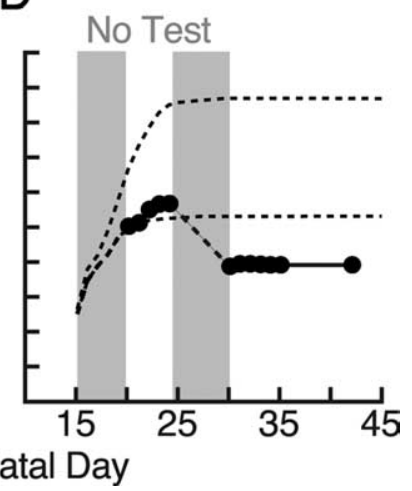

Figure 6. Interaction between induction and consolidation before P25. $\boldsymbol{A}, \boldsymbol{B}$, OKT testing experience from P15 to P19 (A) resulted in an enhancement that was evident at P25-P30, but that subsequently decayed (P72 is plotted). When testing commenced at P20 and continued uninterrupted until P30 (B), the resulting enhancement was smaller, but it endured. These data show that the decay in $\boldsymbol{A}$ was not attributable to a failure to reach maximum, and that the requirement for testing to consolidate enhancement starts between $\mathrm{P} 20$ and P25. C, Testing from P15 to P19 with a break in testing until P30 revealed no enhancement at P72, showing that testing from P20 to P25 in $\boldsymbol{A}$ was temporarily preventing decay (Fig. 4). The dashed line is the "expected" profile from $\boldsymbol{A}$. $\boldsymbol{D}$, Testing experience from P20 to P24 produced a small enhancement (as in $\boldsymbol{B}$ ), but a subsequent 5 d interruption in testing resulted in a decrease to below the baseline of naive animals.

animals with testing from P15 could discriminate between fields of smaller moving dots $\left(0.07^{\circ}\right)$ than animals with testing initiated at P25 $\left(0.35^{\circ}\right)(t=15.0 ; p<0.0001 ; \mathrm{df}=5)$. Thus, animals with enhanced OKT as the result of daily testing from P15 were generally augmented in their ability to use higher SFs when they were present in moving stimuli.

In the last set of experiments, we set out to dissociate the timing of the OKT testing experience necessary for induction and consolidation of enhancement. Figure $6 A$ shows that the regular testing of animals $(n=3)$ from P15 to P19 resulted in enhanced function that appeared to be stabilized by subsequent testing from P25 to P30; however, the enhancement was not maintained into adulthood. In comparison, although a group $(n=2)$ with OKT testing experience from P20 to P25 induced less enhancement (Fig. $6 \mathrm{~B}$ ), continued testing through P30 was able to maintain the enhancement $(t=2.44 ; p=0.025 ; \mathrm{df}=18)$. Thus, daily testing from P20 to P30, not just from P25 to P30 (as might have been surmised from the data in Fig. $3 A, B$ ), was required to consolidate and maintain enhanced responses beyond juvenile life. In addition, Figure $6 B$ shows that the decay in Figure $6 A$ was not attributable to a failure to reach maximal enhancement. In contrast to the period from P20 to P25, P15-P19 was important for induction, but not for consolidation and maintenance. The importance of continuous daily testing was highlighted by experiments in which a period without OKT testing experience from P25 to P30 was combined with testing from P15 to P19 (Fig. 6C) $(n=4)$ or P20 to P25 (Fig. $6 D)(n=3)$. In both situations, the lack of continued daily testing resulted in a reduction of enhancement. In the case of animals tested from P20 to P25, however, the interruption resulted in thresholds well below those in experimentally naive animals $(t=8.15 ; p<0.0001$; df $=22)$. Thus, stimulating plasticity from P20 to $\mathrm{P} 25$, and not following it with repeated testing until P30, leads to a loss of function. This indicated that inductive plasticity is present from P15 to P19, consolidative plasticity is present from P25 to P30, and P20-P25 has elements of both, and that inductive and consolidative processes may interact dynamically during this time.

Absolute monocular directionality of OKT [i.e., only temporal-nasal movement of the stimulus drove OKT through an eye (Douglas et al., 2005)] was maintained in all experiments.

\section{Discussion}

Our results support three main conclusions. (1) Experience- and visual cortexdependent visual plasticity commences in rats at eye opening. (2) Plasticity is manifested in the absence of visual deprivation, as enhancement of visual function, stimulated by relatively short periods of daily visuomotor experience amid otherwise normal visual experience. (3) Properties of the plasticity change over time in distinct but overlapping phases of "induction" and "consolidation."

\section{Experience-dependent enhancement of vision from eye opening}

Previous studies (Harwerth et al., 1990; Smith and Trachtenberg, 2007) have reported that visual deprivation from infancy can lead to visual abnormalities, indicating that developmental plasticity of vision is present in the rat before the critical period for ocular dominance plasticity (Stafford, 1984; Fagiolini et al., 1994; Guire et al., 1999). Our results complement and significantly augment the evidence for plasticity of vision in infancy, by showing that OKT testing experience from eye opening leads to an enhancement of the OKT SF threshold and enhanced perception of moving visual stimuli. The unique methodology of our experiments was also able to demonstrate that plasticity underlying enhancement has distinct phases of induction (the process of experientially enhancing function) and consolidation (the process of experientially stabilizing enhanced function such that it is maintained).

During the induction phase, the day-to-day experience of measuring OKT was found to increase the SF threshold of OKT (Fig. 1), with peak efficacy in late infancy and a profile skewed toward younger ages (Fig. $2 B$ ). The period of inductive plasticity overlaps (Fig. 2A) the period over which experienceindependent OKT is still maturing, suggesting that the ability to enhance function requires that tracking behavior is immature, and that the mechanism of enhancement may involve the augmentation of an intrinsic developmental process. In addition, that visual stimulation and motor activation alone only generate limited enhancement suggests that either referential feedback 
from the head and neck movements or the reduction in retinal slip during tracking is integral to the experience of inducing maximal enhancement. Furthermore, because the maximum daily increase in enhancement (supplemental Fig. 3C, available at www.jneurosci.org as supplemental material) is 1 or $2 \mathrm{~d}$ after the peak of induction (Fig. $2 \mathrm{~B}$ ), it suggests that like ocular dominance plasticity (Frank et al., 2001), a sleep cycle may be necessary to propagate enhancement.

Although we found no evidence that daily testing of OKT after P25 contributed to the induction of enhancement, such experience was a critical variable in the longevity of enhanced function, being both necessary and sufficient to consolidate enhancement so that it is maintained into adulthood (Fig. $3 A, B$ ). The iterative nature of the consolidating experience was highlighted by the result that interrupting testing for as little as $1 \mathrm{~d}$ between P25 and P30, or changing the stimulation parameters after P25, was sufficient to trigger a decline of the enhancement (Fig. 4). These data strongly suggest that juvenile OKT testing experience does not enable new function, but it reinforces function already established in infancy. In addition, notwithstanding the evidence that the induction and consolidation of OKT enhancement are distinct processes, they appear to interact dynamically between P20 and P25 (Fig. 6), because testing OKT between P20 and P25, without continued daily testing through $\mathrm{P} 30$, resulted in a loss of function to a level below that of animals with no testing experience at all (Fig. 6D).

It is worth noting that we have previously tested mouse OKT from eye opening (Prusky et al., 2004) and found no evidence that the testing experience facilitated an enhancement of function. Together with results of the present study, these data indicate that experience-dependent enhancement of visual function as the result of testing OKT from eye opening is thus far limited to rats.

\section{Visual cortex-dependent plasticity}

There are many potential sites for the cellular changes associated with the visual plasticity we report here; however, we were motivated to investigate whether circuits in visual cortex played a role, in part because other work has shown that an adult form of experience-dependent plasticity of OKT is dependent on visual cortex (Prusky et al., 2006). In the present study, we found that visual cortex ablation and inactivation specifically blocked the experience-dependent induction of OKT enhancement (Fig. $1 B, C)$ and that enhanced thresholds in adulthood were abolished with V1 lesions or silencing (Fig. 3C,D). Together, these data indicated that both the induction and maintenance of enhanced OKT are dependent on the integrity of visual cortex. Our finding that enhancement of OKT is accompanied by the ability to use higher SFs in visual motion perception (Fig. $5 B$ ) also supports a role for visual cortex, because the visual cortex has access to SFs $>0.5 \mathrm{c} / \mathrm{deg}$ (Girman et al., 1999; Prusky et al., 2000a) and is thus capable through descending projections (Natal and Britto, 1988; Schmidt et al., 1993; Shintani et al., 1999) of supplementing the inherently low-SF OKT circuitry (Grasse et al., 1984; Simpson, 1984; Grasse and Cynader, 1988; Distler and Hoffmann, 1992; Masson et al., 2001; Hoffmann et al., 2002). In addition, the finding that enhanced OKT thresholds are largest in binocular visual field (Fig. $5 \mathrm{~A}$ ) also implicates visual cortex, because binocular interactions are most conspicuous there.

\section{Normal function of plasticity}

The most parsimonious interpretation of our results is that we have functionally defined a developmental period of experience- dependent plasticity for motion vision that precedes plasticity for form vision. There is precedent for this notion in a study by Daw et al. (1978), in which the negative effects on the directional tuning of cells in visual cortex after directional deprivation rearing were shown to precede, and be separate from, the effects of monocular deprivation on ocular dominance. In addition, recent work by Li et al. (2006) has reported that visual experience very early in postnatal life is necessary for the development of direction selectivity. Thus, the OKT experience that our animals received from eye opening may have provided enriched experience with moving visual stimuli, which specifically enhanced motionrelated tuning properties of visual cortex cells. This would be consistent with our finding that SF thresholds for discriminating moving, but not static, stimuli were enhanced in animals with OKT testing experience from eye opening. Although the plasticity may enable adaptive visuomotor function, engaging it inappropriately could also have clinically nonadaptive implications, because selective visual experience around the transition between induction and consolidation can result in a significant loss of function (Fig. 6).

Another possibility is that the plasticity is linked to the experience-dependent development of image stabilization. Dark- and stroboscopic-rearing experiences are known to produce spontaneous nystagmus (Harris and Cynader, 1981; Melvill Jones et al., 1981); however, that we find changes in visual motion perception (Fig. $5 B$ ), and that exposing developing animals to enriched self motion did not enhance OKT thresholds, speaks against involvement of the corrective, self-motion system. Plasticity of visual pursuit may be better candidate. In the fly, for example, the object tracking system displays experiencedependent plasticity, whereas the image stabilization system does not (Geiger and Poggio, 1975). It is possible, therefore, that rats have a similar separation of functions, with a fixed subcortical pathway for general image stabilization and a plastic cortical pathway for controlling the head and neck movements necessary for visual tracking. Interestingly, short-latency voluntary tracking of visual field shifts involve extrastriate visual cortex (Masson et al., 2001), and we have shown an enhancement in the SF sensitivity of dot-motion coherence (Douglas et al., 2006), a task associated with function of extrastriate cortex (Newsome et al., 1985).

\section{Cellular mechanisms of plasticity}

There are parallels between the developmental visual plasticity we characterized here and at least two electrophysiologically manifested forms of visual plasticity that have been linked with specific cellular events in visual cortex. Jiang et al. (2007) report that synaptic plasticity in the murine visual cortex is developmentally regulated, with plasticity in layer IV being lost by P20, and plasticity in layer II/III continuing into juvenile life. That timing of the loss of layer IV plasticity is correlated with the transition from inductive to consolidative plasticity in our study suggests that the ability to induce OKT enhancement could depend on plasticity in layer IV, and the process of consolidating enhanced responses linked to synaptic changes in layer II/III. In addition, our findings that OKT enhancement depends on specific stimulus conditions and that the testing conditions used to induce enhancement are necessary for its consolidation are indicative of stimulus-selective response potentiation, a form of grating-induced, NMDA receptor-dependent cortical plasticity (Frenkel et al., 2006). We are reminded, however, that causally linking visual physiology with visual function has historically proven to be a challenge. However, the characterization of a physiologically relevant form 
of developmental visual plasticity in which the experiential and temporal variables can be extended across different levels of analysis should make the endeavor in this case more tractable.

\section{References}

Daw NW, Berman NE, Ariel M (1978) Interaction of critical periods in the visual cortex of kittens. Science 199:565-567.

Distler C, Hoffmann KP (1992) Early development of the subcortical and cortical pathway involved in optokinetic nystagmus: the cat as a model for man? Behav Brain Res 49:69-75.

Douglas RM, Alam NM, Silver BD, McGill TJ, Tschetter WW, Prusky GT (2005) Independent visual threshold measurements in the two eyes of freely moving rats and mice using a virtual-reality optokinetic system. Vis Neurosci 22:677-684.

Douglas RM, Neve A, Quittenbaum JP, Alam NM, Prusky GT (2006) Perception of visual motion coherence by rats and mice. Vis Res 46:2842-2847.

Fagiolini M, Pizzorusso T, Berardi N, Domenici L, Maffei L (1994) Functional postnatal development of the rat primary visual cortex and the role of visual experience: dark rearing and monocular deprivation. Vis Res 34:709-720.

Frank MG, Issa NP, Stryker MP (2001) Sleep enhances plasticity in the developing visual cortex. Neuron 30:275-287.

Frenkel MY, Sawtell NB, Diogo AC, Yoon B, Neve RL, Bear MF (2006) Instructive effect of visual experience in mouse visual cortex. Neuron 51:339-349.

Geiger G, Poggio T (1975) The orientation of flies towards visual patterns: On the search for the underlying interactions. Biol Cybern 19:39-54.

Giffin F, Mitchell DE (1978) The rate of recovery of vision after early monocular deprivation in kittens. J Physiol 274:511-537.

Girman SV, Sauvé Y, Lund RD (1999) Receptive field properties of single neurons in rat primary visual cortex. J Neurophysiol 82:301-311.

Grasse KL, Cynader MS (1988) The effect of visual cortex lesions on vertical optokinetic nystagmus in the cat. Brain Res 455:385-389.

Grasse KL, Cynader MS, Douglas RM (1984) Alterations in response properties in the lateral and dorsal terminal nuclei of the cat accessory optic system following visual cortex lesions. Exp Brain Res 55:69-80.

Guire ES, Lickey ME, Gordon B (1999) Critical period for the monocular deprivation effect in rats: assessment with sweep visually evoked potentials. J Neurophysiol 81:121-128.

Harris LR, Cynader M (1981) The eye movements of the dark-reared cat. Exp Brain Res 44:41-56.

Harwerth RS, Smith EL 3rd, Crawford ML, von Noorden GK (1990) Behavioral studies of the sensitive periods of development of visual functions in monkeys. Behav Brain Res 41:179-198.

Heffner RS, Heffner HE (1992) Visual factors in sound localization in mammals. J Comp Neurol 317:219-232.

Hoffmann KP, Bremmer F, Thiele A, Distler C (2002) Directional asymmetry of neurons in cortical areas MT and MST projecting to the NOT-DTN in macaques. J Neurophysiol 87:2113-2123.

Hubel DH, Wiesel TN (1970) The period of susceptibility to the physiological effects of unilateral eye closure in kittens. J Physiol 206:419-436.

Jiang B, Treviño M, Kirkwood A (2007) Sequential development of longterm potentiation and depression in different layers of the mouse visual cortex. J Neurosci 27:9648-9652.

Kolb B, Gibb R (1991) Environmental enrichment and cortical injury: behavioral and anatomical consequences of frontal cortex lesions. Cereb Cortex 1:189-198.

Lehmann H, Clark BJ, Whishaw IQ (2007) Similar development of cued and learned home bases in control and hippocampal-damaged rats in an open field exploratory task. Hippocampus 17:370-380.

Li Y, Fitzpatrick D, White LE (2006) The development of direction selectivity in ferret visual cortex requires early visual experience. Nat Neurosci 9:676-681.

Masson GS, Busettini C, Yang DS, Miles FA (2001) Short-latency ocular following in humans: sensitivity to binocular disparity. Vis Res 41:3371-3387.

McGill TJ, Douglas RM, Lund RD, Prusky GT (2004) Quantification of spatial vision in the Royal College of Surgeons rat. Invest Ophthalmol Vis Sci 45:932-936.

Melvill Jones G, Mandl G, Cynader M, Outerbridge JS (1981) Eye oscillations in strobe reared cats. Brain Res 209:47-60.

Natal CL, Britto LR (1988) The rat accessory optic system: effects of cortical lesions on the directional selectivity of units within the medial terminal nucleus. Neurosci Lett 91:154-159.

Newsome WT, Wurtz RH, Dürsteler MR, Mikami A (1985) Deficits in visual motion processing following ibotenic acid lesions of the middle temporal visual area of the macaque monkey. J Neurosci 5:825-840.

Paxinos G, Watson C (1998) The rat brain in stereotaxic coordinates, Ed 4. San Diego: Academic.

Prusky GT, Douglas RM (2008) Measuring vision in the awake behaving mouse. In: Eye, retina, and visual system of the mouse (Chalupa LM, Williams RL, eds) pp 107-117. Cambridge, MA: MIT.

Prusky GT, Ramoa AS (1999) Novel method of chronically blocking retinal activity. J Neurosci Methods 87:105-110.

Prusky GT, West PW, Douglas RM (2000a) Behavioral assessment of visual acuity in mice and rats. Vis Res 40:2201-2209.

Prusky GT, West PW, Douglas RM (2000b) Experience-dependent plasticity of visual acuity in rats. Eur J Neurosci 12:3781-3786.

Prusky GT, Alam NM, Beekman S, Douglas RM (2004) Rapid quantification of adult and developing mouse spatial vision using a virtual optomotor system. Invest Ophthalmol Vis Sci 45:4611-4616.

Prusky GT, Alam NM, Douglas RM (2006) Enhancement of vision by monocular deprivation in adult mice. J Neurosci 26:11554-11561.

Schmidt M, Zhang HY, Hoffmann KP (1993) OKN-related neurons in the rat nucleus of the optic tract and dorsal terminal nucleus of the accessory optic system receive a direct cortical input. J Comp Neurol 330:147-157.

Shintani T, Hoshino K, Meguro R, Kaiya T, Norita M (1999) A light and electron microscopic analysis of the convergent retinal and visual cortical projections to the nucleus of the optic tract (NOT) in the pigmented rat. Neurobiology 7:445-660.

Simpson JI (1984) The accessory optic system. Annu Rev Neurosci 7:13-41. Smith SL, Trachtenberg JT (2007) Experience-dependent binocular competition in the visual cortex begins at eye opening. Nat Neurosci 10:370-375.

Smith AL, Cordery PM, Thompson ID (1995) Manufacture and release characteristics of Elvax polymers containing glutamate antagonists. J Neurosci Methods 60:211-217.

Smith AL, Parsons CH, Lanyon RG, Bizley JK, Akerman CJ, Baker GE, Dempster AC, Thompson ID, King AJ (2004) An investigation of the role of auditory cortex in sound localization using muscimol-releasing Elvax. Eur J Neurosci 19:3059-3072.

Stafford CA (1984) Critical period plasticity for visual function: definition in monocularly deprived rats using visually evoked potentials. Ophthalmic Physiol Opt 4:95-100.

Wiesel TN, Hubel DH (1963) Single-cell responses in striate cortex of kittens deprived of vision in one eye. J Neurophysiol 26:1003-1017. 